\title{
ESTUDO CLÍNICO COMPARATIVO ENTRE ANASTOMOSE COLOCÓLICA COM ANEL ANASTOMÓTICO BIOFRAGMENTÁVEL E COM SUTURA MANUAL NÃO ABSORVÍVEL
}

\author{
A CLINICAL TRIAL TO COMPARE COLONIC ANASTOMOSIS WITH \\ BIOFRAGMENTABLE ANASTOMOTIC RING AND MANUAL SUTURE
}

\author{
Sarhan Sydney Saad, ACBC-SP ${ }^{1}$ \\ Delcio Matos ${ }^{2}$
}

\begin{abstract}
RESUMO: A anastomose com sutura é método consagrado, apresentando desvantagens teóricas representadas pelo trauma tecidual, edema e alteração da microcirculação. Além disso, o fio de sutura, sendo corpo estranho, determina reação inflamatória, propiciando a aderência e a proliferação de bactérias, assim como de células neoplásicas que seriam evitadas ao se usar método de anastomose sem sutura. A procura por método de anastomose em que não se utilizasse fio de sutura vem sendo realizada desde o início deste século. Com o advento das novas aquisições tecnológicas foi desenvolvido método de anastomose sem sutura através do anel biofragmentável. Esse anel sofre desintegração por hidrólise, sendo eliminado com a evacuação, não permanecendo corpo estranho na anastomose. Esse instrumento permite a realização de anastomose sem sutura, por meio da compressão das paredes intestinais justapostas. O objetivo desse estudo prospectivo e casualizado, foi o de comparar os resultados clínicos e endoscópicos de anastomoses colocólicas, eletivas e de baixo risco, realizadas com anel biofragmentável e com fio de sutura não absorvível. Casuística e Métodos - A casuística foi composta por 36 doentes, divididos em dois grupos de 18, que foram denominados Grupo I (anel biofragmentável ) e Grupo II (sutura com fio não absorvível ), constituídos de doentes comparáveis. Resultados - Os parâmetros de avaliação do período intra-operatório, revelaram que a anastomose com anel biofragmentável despende menor tempo para sua realização. Não se detectou diferença estatisticamente significante entre os dois grupos com relação à incidência de complicações pós-operatórias, mortalidade e avaliação clínica ambulatorial. A incidência de deiscência anastomótica só ocorreu no Grupo I. A análise endoscópica da anastomose no período pós-operatório não demonstrou diferença quanto à perviedade, contratilidade, elasticidade e grau de epitelização. O Grupo II apresentou maior incidência de fio de sutura na anastomose em relação ao Grupo I sendo esta diferença estatística significante. Conclusões - $\mathrm{O}$ anel biofragmentável permite a realização de anastomose de colo mais rápida do que aquela realizada com fio de sutura. Este método de anastomose determinou, na análise global da complicações clínicas, resultados semelhantes ao uso de fio de sutura. O método de anastomose sem sutura apresentou o inconveniente de determinar maior incidência de deiscência anastomótica e como vantagem estabeleceu presença de corpo estranho na anastomose em menor porcentagem.
\end{abstract}

Unitermos : Colo; Anastomose cirúrgica; Técnicas de sutura.

\section{INTRODUÇÃO}

O estudo das anastomoses intestinais se constitui em capítulo de grande importância, pois os índi- ces de deiscência referidos pelos autores, refletem a ausência de um método de anastomose. A anastomose decorrente do tratamento cirúrgico das doenças de cólon e reto apresenta índices de complicações pós-

1. Professor Adjunto - Doutor da Disciplina de Gastroenterologia Cirúrgica da Universidade Federal de São Paulo - Escola Paulista de Medicina.

2. Professor Adjunto - Livre-docente da Disciplina de Gastroenterologia Cirúrgica da Universidade Federal de São Paulo - Escola Paulista de Medicina.

Recebido em 28/10/99

Aceito para publicação em 23/3/2000

Trabalho realizado na Universidade Federal de São Paulo - Escola Paulista de Medicina - Hospital São Paulo. 
operatórias, principalmente no tocante à deiscência, significativos.

A anastomose ideal pressupõe uma união perfeita para a passagem do conteúdo intestinal através de método seguro, com índices mínimos de complicação, mantendo as bordas intestinais com fluxo sangüíneo adequado e sem tensão, com mínimo trauma e ausência de estenose..$^{1-4}$

Entre as técnicas disponíveis para união de bordas intestinais destaca-se a sutura, que pode ser realizada em um ou dois planos. Halsted ${ }^{5}$ descreve a anastomose em um plano, através de agulhas atraumáticas e fio inabsorvível, ressaltando a inclusão da submucosa, pois experimentalmente demonstrou ser esta a camada mais resistente da parede intestinal. Os pontos devem distar $3 \mathrm{~mm}$ entre si e estar situados a $2 \mathrm{~mm}$ da borda intestinal ${ }^{6}$. Faria ${ }^{7}$, em nosso meio, efetuou trabalho experimental em cães onde comparou a sutura extramucosa em plano único, com sutura em dois planos, que revelou uma coaptação das bordas intestinais mais anatômica, com menor processo inflamatório e melhor vascularização dos tecidos, concluindo que a sutura em um plano é superior a em dois planos.

A anastomose sem sutura, sem pontos ou grampos, permite a manutenção de duas alças intestinais seccionadas até que ocorra a cicatrização da ferida operatória. Este tipo de anastomose é representado pelos instrumentos de compressão cuja ação se baseia em manter as bordas intestinais unidas por meio de aparelho mecânico, devendo conservar este contato até que a cicatrização da ferida adquira força o suficiente para manter esta união de per si, sendo então eliminado, resultando em cicatriz intestinal sem corpo estranho.

Hardy Jr. et al. ${ }^{8}$, fazendo uso de modernas aquisições bioquímicas e de bioengenharia, desenvolveram o anel anastomótico biofragmentável (AAB). Este instrumento é formado por dois componentes idênticos, constituídos por ácido poliglicólico ( $87,5 \%$ ) e sulfato de bário ( $12,5 \%$ ), que se inter-relacionam, de maneira a se fixarem um ao outro. Está disponível em calibres de $25 \mathrm{~mm}, 28 \mathrm{~mm}, 31 \mathrm{~mm}$ e $34 \mathrm{~mm}$, com abertura de $6 \mathrm{~mm}$ entre os segmentos, podendo apresentar duas medidas ( $1,5 \mathrm{~mm}$ e $2 \mathrm{~mm}$ ) após a oclusão dos mesmos. As bordas intestinais devem ser fixadas em torno do orifício central de cada um dos seus componentes, através de sutura em bolsa. Após a oclusão dos seus segmentos, os componentes não mais retornam à posição original, realizando desta forma anastomose por inversão, sem determinar necrose de todo tecido interposto. Após um período variável de duas a três semanas, este instrumento se fragmenta por hidrólise, sendo os produtos de sua divisão eliminados pelas fezes. Os estudos experimentais e clínicos com o uso deste tipo de anastomose demonstraram sua segurança através dos bons resultados obtidos. $^{9-13}$

O objetivo do presente estudo foi comparar os resultados clínicos e endoscópicos, no período pós-operatório intra-hospitalar e durante o acompanhamento ambulatorial, entre a anastomose colocólica com a utilização do anel anastomótico biofragmentável e a sutura com fio não absorvível, em doentes submetidos à cirurgia eletiva e de baixo risco.

\section{PACIENTES E MÉTODOS}

Incluíram-se neste estudo doentes portadores de doenças colônicas cujo tratamento exigisse anastomose colocólica eletiva. Como fatores de exclusão considerou-se a presença de carcinomatose, ou qualquer outra doença terminal, doenças concomitantes, tais como hipertensão e diabetes, estado de descompensação, desnutrição grave, doença inflamatória intestinal, arteriopatia sistêmica grave, enterocolite actínica, uso de drogas imunossupressoras ou preparo inadequado de colo durante o ato operatório.

Neste trabalho, foram estudados prospectivamente 36 doentes, atendidos de maneira consecutiva, pela disciplina de Gastroenterologia Cirúrgica da Escola Paulista de Medicina - Universidade Federal de São Paulo, no período compreendido entre agosto de 1992 e maio de 1994. Esta pesquisa foi aprovada pela Comissão de Pós-graduação da Disciplina de Gastroenterologia Cirúrgica e pela Comissão de Ética do Hospital São Paulo - Escola Paulista de Medicina - Universidade Federal de São Paulo, devendo os doentes assinar termo de consentimento.

Os doentes foram divididos em dois grupos de 18 componentes. Grupo I: doentes nos quais se realizou anastomose colocólica com anel anastomótico biofragmentável. Grupo II: doentes nos quais se realizou anastomose colocólica, em plano único seromuscular, com fio de sutura monofilamentar não absorvível e pontos separados.

Houve predomínio do sexo masculino, tanto no Grupo I como no II, o que correspondeu a $66,7 \%$ e $72,2 \%$ dos doentes, respectivamente. Não houve diferença estatística entre os grupos, com relação à distribuição do sexo e à média de idade. A indicação de cirurgia entre os doentes do Grupo I foi a de reconstrução do trânsito intestinal, após a realização de colostomia em alça esquerda (nove doentes) por ferimento de reto, colostomia direita (seis doentes) e neoplasia maligna de colo esquerdo (três doentes). No Grupo II a cirurgia foi indicada para reconstrução do trânsito após realização de colostomia direita (oito doentes), colostomia esquerda (sete doentes) e neoplasia maligna do colo esquerdo (três doentes).

Todos os doentes incluídos realizaram estudo bioquímico padronizado pelo protocolo de pesquisa, que teve como objetivo a detecção de anormalidades capazes de refletir alterações de seu estado clínico, não se detectando diferença estatisticamente significante entre os dois grupos.

Os doentes foram avaliados, no período pré-operatório, quanto ao seu estado nutricional , através do peso, medidas antropométricas e parâmetros bioquímicos. O mesmo número de doentes do Grupo I e do Grupo II (14$77,8 \%$ ) foi considerado eutrófico e quatro (22\%) com desnutrição leve.

Os doentes foram classificados pelo risco cirúrgico pré-operatório, em função das doenças preexistentes, segundo a Associação Americana de Anestesiologistas. ${ }^{14} \mathrm{De}$ acordo com essa classificação, apresentavam risco classe I $12(66,7 \%)$ doentes do Grupo I, o mesmo ocorrendo com nove $(50 \%)$ do Grupo II. Como risco classe II, foram qualificados seis $(33,3 \%)$ doentes do Grupo I e nove $(50,0 \%)$ do Grupo II. 
Todos os doentes foram submetidos a preparo de colo, com início dois dias antes da cirurgia, que consistiu na limpeza mecânica do colo e no uso de antimicrobianos profilaticamente.

Realizou-se um total de 36 anastomoses. O cirurgião, assim como o primeiro auxiliar, sempre foram os mesmos em todas as cirurgias realizadas nos doentes deste estudo. A anastomose colocólica esquerda foi a mais freqüente, correspondendo a $12(66,7 \%)$ e dez $(55,6 \%)$ doentes, enquanto a colocólica direita foi realizada em seis $(33,3 \%)$ e oito $(44,4 \%)$ doentes, respectivamente nos Grupos I e II. A análise estatística não detectou diferença significante entre os grupos, com relação ao tipo de anastomose realizada.

Nos segmentos intestinais a serem anastomosados, fazia-se a medida do diâmetro das bordas intestinais a serem unidas. Outra mensuração realizada foi a da espessura da parede do colo, por meio de paquímetro. Essas medidas tinham como objetivo a escolha adequada do anel anastomótico biofragmentável, com relação ao seu diâmetro e ao intervalo após o fechamento. A escolha do tipo de anastomose era efetuada por randomização através de 36 envelopes, que continham em seu interior a indicação da forma de anastomose. Este procedimento só foi realizado após o cirurgião constatar que ambas as técnicas eram executáveis e igualmente apropriadas ao doente.

$\mathrm{O}$ anel anastomótico biofragmentável utilizado neste estudo foi o Valtrac ${ }^{\circledR}$, (Figura 1). Iniciava-se a contagem do tempo de anastomose quando se aplicava a pinça própria (D\&G no 514) para a realização de sutura em bolsa, utilizando-se fio monofilamentar absorvível de poligliconato 3-0 com agulha reta. Com o auxílio do primeiro e segundo dedos, fazia-se a compressão dos segmentos do anel, de maneira firme e contínua, até se obter o ruído característico que indica a oclusão do anel (Figura 2). Nesse ponto encerrava-se a contagem do tempo. A anastomose era testada quanto à sua continência, por meio de manobras de compressão dos segmentos cólicos proximais e distais. $\mathrm{O}$ anel calibre $31 \mathrm{~mm}$ e intervalo $1,5 \mathrm{~mm}$ foi utilizado em $11(61,1 \%)$ doentes, o de calibre $28 \mathrm{~mm}$ e intervalo $2,0 \mathrm{~mm}$ em quatro $(22,2 \%)$ doentes e o de calibre $34 \mathrm{~mm}$ e intervalo de $2,0 \mathrm{~mm}$ em três $(16,7 \%)$ doentes.

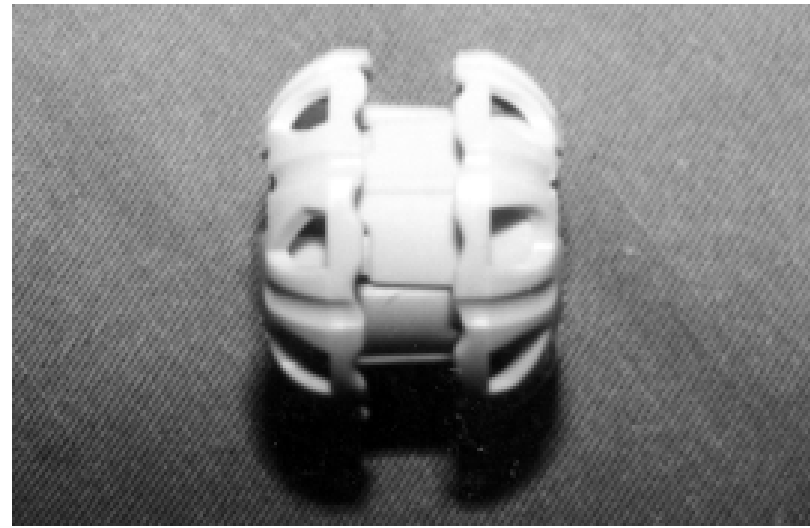

Figura 1 - Anel anastomótico biofragmentável — vista lateral.

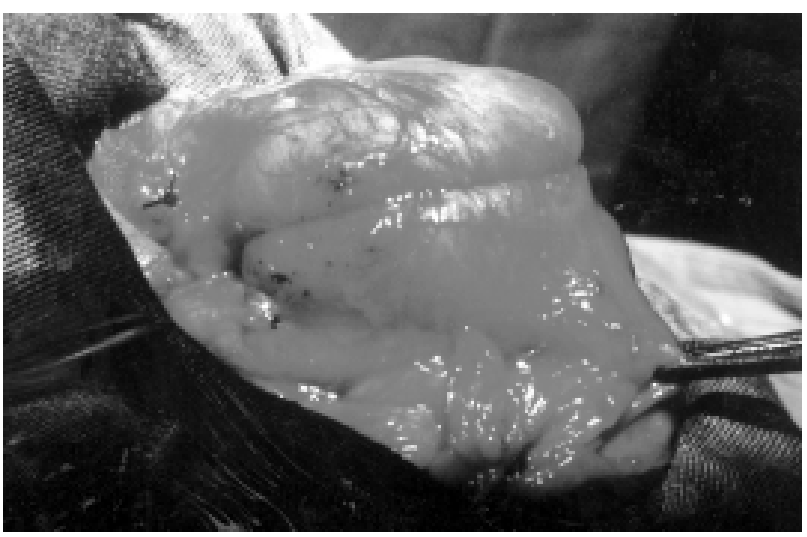

Figura 2 - Anastomose colo-cólica com anel biofragmentável.

A anastomose com fio foi realizada com sutura monofilamentar não absorvível, de polipropileno 3-0, atraumático, com agulha de corte transversal cilíndrico, de $2,5 \mathrm{~cm}$. A anastomose foi sempre término-terminal, realizada por meio de pontos separados, em plano único seromuscular, tomando-se o cuidado de incorporar na sutura a camada submucosa. Os pontos se dispunham paralelamente na linha de anastomose, distando entre si cerca de 2 a $3 \mathrm{~mm}$. Determinou-se o início da contagem de tempo ao se dar o primeiro ponto e seu final ao término do procedimento. A continência foi testada de maneira semelhante ao outro tipo de anastomose, verificando-se também a perviedade, por meio de manobras digitais.

Em todos os doentes realizou-se a drenagem da cavidade abdominal com dreno de borracha laminar, tipo Penrose, posicionado próximo à anastomose e exteriorizado por contra-abertura na parede abdominal.

Utilizaram-se os seguintes parâmetros clínicos no cotejamento dos grupos: período de íleo adinâmico e início da dieta por via oral, sinais clínicos de deiscência anastomótica e mortalidade pós-operatória, tempo de hospitalização e avaliação clínica e endoscópica durante o período de acompanhamento ambulatorial.

A colonoscopia pós-operatória foi efetuada com aparelho de videocolonoscopia GFV101, precedido de preparo mecânico do colo com manitol a $10 \%$ e com sedação durante o mesmo. Os exames foram realizados por um mesmo examinador que sabia a que grupo pertencera $\mathrm{o}$ doente, sendo registrados em fita de videocassete. A conclusão dos resultados foi obtida por meio da revisão detalhada dos registros dos exames. A avaliação colonoscópica baseou-se em cinco parâmetros: perviedade classificada como: excelente quando o diâmetro da anastomose correspondia ao diâmetro do colo; boa na presença de esporão que não interferia com o trânsito fecal; regular, ao se constatar que o aparelho passava ajustado à anastomose, e má quando não se ultrapassava a mesma. A elasticidade era pesquisada pela insuflação de ar no nível da anastomose e foi classificada como presente ou ausente. A contratilidade foi pesquisada pela visibilização da onda contrátil no nível da anastomose, e na epitelização se estudava a mucosa cólica no nível da anastomose, anotando altera- 
ções tais como ulcerações e presença de fibrina. Os fios de sutura e granulomas eram anotados como presentes ou ausentes.

Objetivando-se avaliar possíveis diferenças entre os dois grupos anteriormente definidos, usou-se o teste não paramétrico de Kruskal-Wallis, que, quando necessário, foi complementado pelo teste de comparações múltiplas, o teste do Qui $\left(\mathbf{X}^{2}\right)$ quadrado e, na presença de restrições de Cochran, usou-se o teste exato de Fisher, assim como o teste de MannWhitney. Em todos os casos fixou-se para rejeição da hipótese de nulidade um valor igual ou menor que 0,05 (5\%).

\section{RESULTADOS}

Em 17 doentes (94,4 \%) do Grupo I e em 14 (77,8\%) do Grupo II, o preparo de colo pôde ser considerado excelente, não havendo diferença estatística significante entre os dois grupos.

O tempo despendido para realização das anastomoses do Grupo I, variou de 7 minutos e 30 segundos a 25 minutos, sendo em média de 14 minutos e 4 segundos. A anastomose com sutura, relativa ao Grupo II, variou de 22 minutos e 30 segundos a 34 minutos, com uma média de 27 minutos e 30 segundos. O tempo de anastomose no Grupo I foi significativamente menor do que no Grupo II.

Os Grupos I e II não diferiram de maneira significativa quanto ao dia pós-operatório para início da dieta por via oral, sendo em média de 2,9 dias para o Grupo I e 2,7 dias para o Grupo II.

A eliminação do anel biofragmentável através da evacuação pôde ser registrada em $15(83,3 \%)$ doentes, sendo que essa ocorrência se deu sem qualquer incômodo. $\mathrm{O}$ anel foi eliminado entre 13 e 28 dias, com média de 20,6 dias.

Dos 36 doentes, $13(36,1 \%)$ apresentaram complicação pós-operatória abdominal. No Grupo I, sete doentes apresentaram nove complicações, correspondendo a índice de morbidade de $38,9 \%$, enquanto que, no Grupo II, ocorreram seis complicações em seis doentes, o que significou um índice de $33,3 \%$. A infecção de ferida cirúrgica foi de grau leve em quatro doentes do Grupo I e em quatro do Grupo II. Foi considerada de grau moderado em um doente de cada grupo. Considerou-se como portador de deiscência de anastomose o doente que apresentou, no curso pós-operatório, fístula, ou seja, comunicação anormal entre o colo e a pele, por meio da drenagem de secreção intestinal pelo dreno de borracha laminar (Penrose).
A fístula foi considerada de alto ou baixo débito em função de sua drenagem diária situar-se acima ou abaixo de $500 \mathrm{ml}$ por dia, respectivamente. ${ }^{15}$ Houve quatro fístulas nos doentes do Grupo I, sendo duas de baixo débito e duas de alto débito. Os primeiros doentes apresentaram curso pós-operatório sem intercorrências. As fístulas de alto débito determinaram a reoperação desses doentes. Em um deles se desfez a anastomose e as bordas cólicas foram exteriorizadas, enquanto no outro se realizou sutura da deiscência e colostomia em alça no colo direito. Obstrução intestinal foi caracterizada em um doente do Grupo II que também necessitou de reoperação. A Tabela 1 demonstra as complicações pós-operatórias abdominais não se detectando diferença estatística entre os Grupos I e II. Não houve mortalidade na presente casuística.

O tempo de hospitalização dos doentes do Grupo I variou de seis a 27 dias, sendo em média 12,4 dias, enquanto que no Grupo II a variação foi de quatro a 24 dias, com média de 10,4 dias, não havendo diferença estatística.

Todos os doentes foram acompanhados em nível ambulatorial, por um período mínimo de três meses e, do ponto de vista de evolução clínica, não se constatou quaisquer complicações da anastomose. Tanto no Grupo I como no Grupo II, a colonoscopia foi realizada em $13(72,2 \%)$ doentes. O tempo decorrido entre a cirurgia e a colonoscopia pós-operatória variou de 49 a 128 dias, sendo em média de 84,8 dias no Grupo I, e variou de 32 a 160 dias, com média de 72,2 dias no Grupo II, não se detectando diferença estatística. A perviedade foi considerada excelente ou boa em $92,3 \%$ nos dois grupos, havendo ocorrência de um $(7,7 \%)$ doente em cada grupo no qual esse parâmetro foi estimado como regular. A contratilidade, assim como a elasticidade, esteve presente em todos os doentes analisados. No exame da epitelização, houve alteração desse critério em somente um $(7,7 \%)$ doente do Grupo I, enquanto nos restantes foi considerada normal. $\mathrm{O}$ estudo da presença de fio de sutura e de granuloma demonstrou que os doentes do Grupo II apresentaram estes parâmetros com uma freqüência superior aos doentes do Grupo I, sendo esta diferença estatisticamente significante (Figuras 3 e 4).

\section{DISCUSSÃO}

Esse estudo teve como finalidade a comparação de dois procedimentos que visam à obtenção de anastomose intestinal. Para sua realização, optou-se pelo modelo

Tabela 1

Complicações pós-operatórias abdominais

\begin{tabular}{l|cc|cc|cr}
\hline & \multicolumn{2}{|c|}{ Grupo I } & Grupo II & & Total \\
\cline { 2 - 7 } & No. de doentes & $\%$ & No. de doentes & $\%$ & No. de doentes & $\%$ \\
\hline Com complicação & 7 & 38,9 & 6 & 33,3 & 13 & 36,1 \\
Sem complicação & 11 & 61,1 & 12 & 66,7 & 23 & 36 \\
\hline Total & 18 & 100,0 & 18 & 100,0 & 100,0 \\
\hline
\end{tabular}




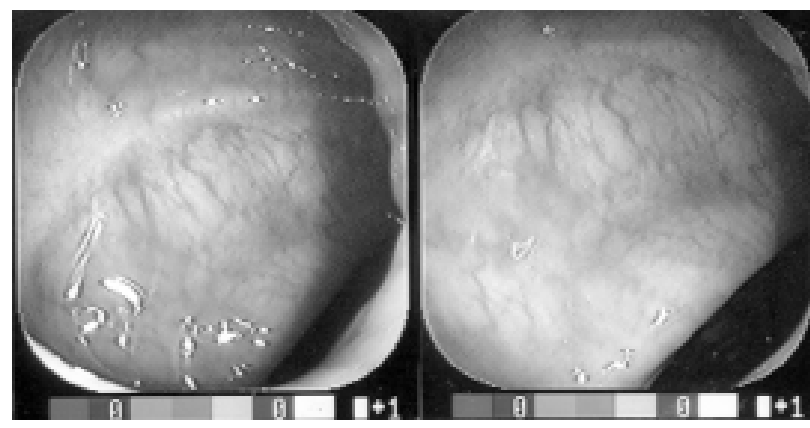

Figura 3 - Exame endoscópico de anastomose colo-cólica com anel bioframentável. Anastomose identificada por linha esbranquiçada.

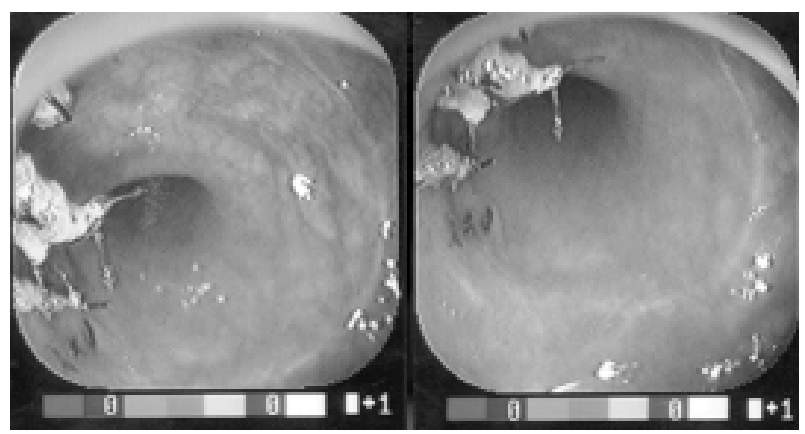

Figura 4-Exame endoscópico de anastomose colo-cólica com sutura. Anastomose identificada por presença de corpo estranho - fio de sutura.

prospectivo, controlado e casualizado. A obtenção de amostras homogêneas que possam ser confrontadas é passo fundamental e para isso a inclusão dos componentes de cada amostra deve ser feita ao acaso. A casuística obedeceu a rígida seleção de doentes, restringindo o tamanho da amostra, constituída por dois grupos comparáveis, compostos por 18 doentes, inseridos na pesquisa de maneira consecutiva. Os doentes dos dois grupos não diferiram estatisticamente quanto à média de idade, sexo, resultados laboratoriais, estado nutricional e risco cirúrgico pré-operatório.

A equipe cirúrgica foi sempre composta pelo mesmo cirurgião e primeiro auxiliar. A fístula após ressecção cólica, tem maior incidência após as anastomoses em colo esquerdo em relação ao direito. ${ }^{16}$ As anastomoses, neste estudo, foram divididas topograficamente em colocólicas direita e esquerda não se demonstrando diferença estatisticamente significante entre os dois grupos.

$\mathrm{O}$ anel biofragmentável representa método de união intestinal por compressão, permitindo a realização de anastomose sem sutura, sendo que, no período crítico da cicatrização, esse instrumento desempenha o papel de meio de sustentação mecânica das alças intestinais a serem unidas. As pesquisas em animais de experimentação, comparando esse procedimento a outros tipos de anastomose, demonstraram a segurança do método. ${ }^{8,13} \mathrm{O}$ bom resulta- do da sutura em bolsa é etapa de fundamental importância, na execução da anastomose com anel, pois ao contrário da anastomose por grampeamento, em que essa sutura é retirada no final do procedimento, no anel ela deverá permanecer de cinco a sete dias, garantindo a estabilidade da anastomose. Dessa forma, toda sutura em bolsa inadequada deverá ser refeita, pois irá, quase certamente, determinar deiscência. ${ }^{17,18}$

Duas avaliações das alças a serem unidas, representadas pela medida do calibre e a determinação da espessura, deverão ser feitas de maneira precisa, porque, caso o anel possua maior diâmetro do que a alça, isso irá determinar laceração de sua mucosa ou serosa. O erro na avaliação do calibre poderá permitir que o anel passe a fazer compressão da parede intestinal, o que de certa forma determinaria alteração da circulação intraparietal, condição que levou alguns autores a abandonarem essa técnica. 11,19,20 Da mesma forma, a falta de exatidão na medida da espessura da alça induzirá à escolha de anel cujo intervalo, após sua oclusão, seja inadequado. Não ocorrerá justaposição serosserosa satisfatória se esse intervalo for muito largo e, se o mesmo for demasiadamente estreito, é possível que não se obtenha a oclusão dos componentes do anel. Nessa última condição, caso se obtenha o fechamento do anel, poderá ocorrer necrose excessiva do tecido interposto, com conseqüente deiscência das bordas intestinais unidas. O fechamento completo dos componentes do anel é outro passo a ser obtido com rigoroso cuidado, visto que, quando se obtém o ruído característico, isso nem sempre significa que o processo está concluído, sendo necessária a confirmação da acoplagem dos segmentos por meio de novas compressões segmentares no instrumento. Não se deve fazer compressão excessiva nessa fase, pelo risco de provocar hematomas na parede da alça, ou mesmo de fragmentar o anel. ${ }^{21}$ Nessa casuística não foram registrados acidentes intra-operatórios durante a execução da anastomose com anel biofragmentável.

A anastomose que utiliza fio de sutura mantém a aposição das alças intestinais a serem unidas até que a cicatrização se complete. Os estudos clínicos prospectivos e randomizados não encontraram diferenças entre os resultados obtidos com a sutura em plano único, em relação aos registrados na sutura em dois planos. ${ }^{21-23} \mathrm{O}$ fio de sutura de configuração monofilamentar e de natureza sintética não absorvível, representado pelo fio polipropileno, é o material que determina menor reação inflamatória, menor risco de infecção e melhor evolução do processo de cicatrização. $^{24,25}$ Experimentalmente são apontadas desvantagens quando da utilização de fio de sutura na execução de anastomose intestinal, as quais, no entanto, não levam a alterações clínicas significantes, a ponto de impedir o uso rotineiro desse material. Estas dizem respeito ao trauma tecidual, alterações da microcirculação, reação inflamatória, predisposição a sépsis anastomótica e implantação de células neoplásicas. ${ }^{26-29} \mathrm{O}$ método de preparo mecânico do colo efetuado nos doentes dessa casuística mostrou-se eficaz, já que, na maioria dos doentes dos dois grupos, o grau de limpeza do colo na cirurgia foi considerado excelente, não existindo diferença significante entre os mesmos. 
A anastomose com anel biofragmentável pode ser realizada mais rapidamente que a sutura manual ou mesmo a mecânica. ${ }^{13,19,21,30}$ Neste estudo o tempo médio despendido para a execução da anastomose com anel biofragmentável foi de 14 minutos. A análise estatística revelou que ocorreu diferença significante entre o tempo de realização da anastomose com anel e com fio, caracterizando-se maior rapidez na execução da anastomose com o primeiro método.

A análise estatística não revelou diferença significante entre os grupos, no tocante à liberação da alimentação por via oral.

A eliminação do anel é referida pelos diferentes autores em período que varia de 16 a 23 dias pós-operatórios, sem lesões da mucosa retal ou desconforto para o doente. ${ }^{9}$ Nos doentes desse estudo, o anel foi eliminado em média 20 dias após a cirurgia, não havendo relato de qualquer sintoma relacionado a esse episódio.

Mesmo não se constatando diferença estatística significante entre o Grupo I e Grupo II em relação à frequiência de complicações pós-operatórias abdominais, houve expressiva diferença entre os grupos quanto à incidência de fístula anastomótica.

A deiscência de anastomose após cirurgia de colo constitui-se na mais grave ocorrência pós-operatória. $\mathrm{O}$ Quadro 1 apresenta os índices de deiscências após o uso de anel biofragmentável. O Quadro 2 contém as freqüências registradas para a deiscências em anastomoses realizadas em plano único seromuscular extramucoso.

\section{Quadro 1}

Freqüência de deiscência em anastomoses realizadas com anel biofragmentável

\begin{tabular}{|c|c|c|}
\hline Autor & $\begin{array}{c}\text { Doentes } \\
\text { No. }\end{array}$ & $\begin{array}{c}\text { Deiscência } \\
(\%)\end{array}$ \\
\hline HARDY Jr. et al. $(1987)^{9}$ & 27 & 0,0 \\
\hline CAHILL et al. (1989) $)^{17}$ & 101 & 2,0 \\
\hline CORMAN et al. (1989) & 222 & 2,7 \\
\hline DYESS et al. $(1990)^{19}$ & 27 & 0,0 \\
\hline LUKKONNEN et al. $(1990)^{20}$ & 20 & 15,0 \\
\hline BUBRICK et al. $(1991)^{10}$ & 395 & 3,0 \\
\hline GULLICHSEN et al. $(1992)^{12}$ & 79 & 2,5 \\
\hline CHUA $(1993)^{21}$ & 20 & 0,0 \\
\hline FORDE et al. $(1993)^{33}$ & 31 & 0,0 \\
\hline WOOD e FROST $(1993)^{34}$ & 47 & 0,0 \\
\hline HABR-GAMA et al. $(1994)^{35}$ & 25 & 4,0 \\
\hline SEOW-CHEN et al. (1994) & 21 & 0,0 \\
\hline KONISHI et al. $(1995)^{31}$ & 40 & 2,5 \\
\hline
\end{tabular}

Os trabalhos comparativos, prospectivos e casualizados que cotejaram o anel biofragmentável com a sutura convencional e com grampeamento não encontraram diferença estatisticamente significante entre os diferentes métodos no tocante à frequiência de fístula anastomótica

\section{Quadro 2}

Freqüência de deiscência em anastomoses realizadas com anel de sutura em plano único seromuscular extramucoso

\begin{tabular}{l|c|c}
\hline Autor & $\begin{array}{c}\text { Doentes } \\
\text { No. }\end{array}$ & $\begin{array}{c}\text { Deiscência } \\
(\%)\end{array}$ \\
\hline BELING (1957) $)^{37}$ & 60 & 0,0 \\
BRONWELL et al. $(1967)^{38}$ & 136 & 0,0 \\
PETERS e STANTEN $(1971)^{39}$ & 175 & 2,9 \\
MANTOVANI et al. $(1975)^{40}$ & 30 & 0,0 \\
ROSENBERG et al. $(1975)^{41}$ & 32 & 0,0 \\
MATHESON et al. $(1985)^{41}$ & 206 & 1,5 \\
CARTY et al. $(1991)^{43}$ & 500 & 2,2 \\
\hline
\end{tabular}

pós-operatória. ${ }^{10,11,12,17,19,35}$ Neste estudo, a freqüência de fístula pós-operatória, na anastomose com sutura convencional está concorde com os índices encontrados na literatura pertinente. $\mathrm{O}$ mesmo não ocorreu com os índices de deiscência obtidos após o uso do anel biofragmentável, os quais se revelaram muito discordantes dos índices registrados pelos autores consultados, mesmo não se utilizando outros recursos diagnósticos na pesquisa dessa complicação. Na análise da incidência de fístula pós-operatória entre os dois método testados, ocorreu diferença expressiva, ou seja, 22,2 \% nos casos em que o anel foi utilizado e ausência dessa complicação nos doentes em que foi efetuada a anastomose com fio de sutura. Mesmo que se considerem somente os casos de fístulas de alto débito $(11,1 \%$ - dois doentes), configurados como situações de extrema gravidade, com imposição de reoperação dos doentes e exposição a maior risco de mortalidade, não é possível deixar de notar a maior freqüência dessa complicação nos doentes do Grupo I. O limitado número de doentes dessa amostra poderia explicar tal fato, pois, hipoteticamente, com número maior de doentes, essa diferença não seria tão marcante.

Na presente casuística não houve mortalidade.

Não houve diferença estatisticamente significante, quanto ao tempo de permanência hospitalar, entre os dois tipos de anastomose, em estudos prospectivos e casualizados. ${ }^{10,11,12,17,19,35}$ Nos doentes analisados nesta pesquisa, o tempo de hospitalização para o Grupo I foi de 12,4 dias e para o Grupo II de 10,4 dias.

Entre os autores que utilizaram o anel biofragmentável existe referência a pequeno número de doentes que evoluíram com estenose anastomótica. ${ }^{9,20,43}$ Nos doentes deste estudo, durante o acompanhamento ambulatorial, não se detectou do ponto de vista clínico indicações de que a anastomose com anel biofragmentável ou com sutura convencional tivessem evoluído com estenose, durante esse período.

Nessa casuística, com o objetivo de fazer avaliação mais acurada da evolução das anastomoses, foi indicado exame colonoscópico no período de acompanhamento ambulatorial. Nos 36 doentes os achados endoscópicos demonstraram que a maioria deles, em ambos os grupos, apresentava bom calibre do colo no nível da anastomose, 
o local da anastomose com boa contração e elasticidade e a epitelização normal. No que se refere à presença de fios de sutura e tecido de granulação, houve diferença estatisticamente significante entre os Grupos I e II.

$\mathrm{O}$ anel bio-fragmentável demonstrou ser um método simples de anastomose e pôde ser efetuado em menor tempo que a sutura com fio não absorvível. A anastomose com anel biofragmentável não determinou alteração no início da dieta por via oral no período pós-operatório, assim como no número de complicações pós-operatórias abdominais quando confrontada com a sutura com fio não absorvível, apesar da maior propensão à deiscência anastomótica. A evolução clínica no período de acompanhamento ambulatorial não demonstrou diferenças entre a anastomose com anel biofragmentável e com fio de sutura não absorvível.

\begin{abstract}
The use of suture in anastomosis presents some theoretical disadvantages that they would be avoided by using sutureless intestinal anastomosis. The biofragmentable ring idealized by Hardy et al.(1985), is composed by polyglycolic acid and barium sulfate. It is constituted by two identical components that are coupled, not returning to the original position after its occlusion. This ring suffers desintegration in 2 to 3 weeks, and is eliminated with evacuation. This device allows a sutureless anastomosis by the compression of intestinal walls. The purpose of this prospective and randomized study was to compare the clinical and endoscopic results in eletive and low risk colonic anastomosis accomplished with biofragmentable anastomosis ring and with non absorbable suture. Material: Thirty-six patients were stratified in two groups of 18 that were denominated Group I (biofragmentable anastomosis ring) and Group II (non absorbable suture). The average age for Group I was 36 years and for Group II was 42. The most frequent surgical indication in both groups was reconstruction of the intestinal flow after colostomy. All patients were submitted to biochemical blood tests, nutritional and surgical risk evaluation. The statistical analysis used for comparison between groups demonstrated that they were homogeneous. Results: Comparative analysis of the intraoperative period parameters revealed that the anastomosis with biofragmentable anastomosis ring is faster ant there are no significant differences regarding intraoperative complications rate, period of ileus, time to reintroduce the oral diet, mortality and long term folloow-up. The incidence of anastomotic leak was higher in the Group I. The endoscopic analysis demonstrate no difference because there was the same results for both groups. There was a significant higher incidence of exposed suture in suture anastomosis when compared to biofragmentable anastomosis ring. Conclusions: It can be concluded that the biofragmentable anastomosis ring allowed the accomplishment of colon anastomosis more quickly, and regarding clinical complications, similar to the use of non absorbable suture. This anastomosis method presented the inconvenience of determining higher incidence of anastomotic leak while as advantage it established presence of exposed suture in the anastomosis in smaller percentage.
\end{abstract}

Key Words: Colon Surgery; Anastomosis; Suture.

\section{REFERÊNCIAS}

1. Maurya, S.D.; Gupta, H.C.; Tewari, A.et al: Double layer versus single layer intestinal anastomosis: a clinical trial. Int. Surg. 1984; 69: 339-40.

2. Orr, N.W.M.: A single-layer intestinal anastomosis. $\mathrm{Br} . J$. Surg 1969; 56: 771-4.

3. Fraser, I. : An historical perspective on mechanical aids in intestinal anastomosis. Surg. Gynecol. Obstet.1982; 155: 566-74.

4. Schrock, T.R.; Deveney, C.W.; Dunphy, J.E.: Factors contributing to leakage of colonic anastomoses. Ann. Surg 1973; 177: 513-8.

5. Halsted, W.S.: Circular suture of the intestine - An experimental study. Am. J. Med. Sci. 1887; 94: 436-61.

6. Gorodiche, J. \& Jourdan, P.: Anastomoses digestives en un plan de suture. Semin. Hôp. Paris 1951; 4: 3740-7.

7. Faria, P.A. J.: Sutura gastrointestinal em plano único extramucoso e em 2 planos, um total e um seromuscular invaginante. Estudo experimental no cão. São Paulo, 1972. 106p. [Tese de Doutoramento - Escola Paulista de Medicina]

8. Hardy JR., T.G.; Pace, W.G.; Maney, J.W. et al: A biofragmentable ring for sutureless bowel anastomosis. An experimental study. Dis. Colon Rectum 1985; 28: 48490.

9. Hardy JR., T.G.; Aguilar, P.S.; Stewart, W.R.C. et al: Initial clinical experience with a biofragmentable ring for sutureless bowel anastomosis. Dis. Colon Rectum 1987; 30: $55-60$

10. Bubrick, M.P.; Corman, M.L.; Cahill, C.J. et al: The BAR Investigational Group - Prospective, randomized trial of the biofragmentable anastomosis ring. Am. J. Surg. 1991; 161: 136-42.

11. Corman, M.L.; Prager, E.D.; Hardy JR., T.G. et al: The Valtrac (BAR) Study Groups - Comparison of the valtrac biofragmentable anastomosis ring with Conventional suture and stapled anastomosis in colon surgery. 
Results of a prospective, randomized clinical trial Dis. Colon Rectum 1989; 32: 183-5.

12. Gullichsen, R.; Havia, T.; Ovaska, J. et al: Colonic anastomosis using the biofragmentable anastomotic ring and manual suture: a prospective, randomized study. Br. J. Surg 1992; 79: 578-80.

13. Matos, D.; Saad, S.S. ; Franceschi JR., O. et al: Estudo experimental comparativo entre anastomose colocólica com sutura manual e com anel biofragmentável. Rev. Assoc. Med. Bras 1993; 39: 201-6.

14. Dripps, R.D.; Lamont, A.; Eckenhoff, J.E.: The role of anesthesia in surgical mortality. J. Am. Med. Assoc. 1961; 178: 261-6.

15. Keighley, M.R.B.: "Intestinal fistulas”. In: Keighley, M.R.B., Williams, N.S. (eds.): Surgery of anus, rectum and colon. 1.ed. Londres: WB Saunders Company Ltd., 1993, vol.2., pp.2013-102.

16. Hoier-Madsen, K.; Hansen, J.B.; Lindenberg, J.: Anastomotic leakage following resection for cancer of the colon and rectum . Acta Chir. Scand. 1975; 141: 304-9.

17. Cahill, C.J.; Betzler, M.; Gruwez, J.A. et al: Sutureless large bowel anastomosis : european experience with the biofragmentable anastomosis ring. Br. J. Surg. 1989; 76 : 344-7.

18. Zederfeldt, B.; Jiborn, H.; Ekelund,G. : Sutureless colonic anastomoses. Langenbecks Arch. Chir. 1990; 375: 181-5, 1990.

19. Dyess, D.L.; Curreri, P.W.; Ferrara, J.J. : A new technique for sutureless intestinal anastomosis . A prospective, randomized, clinical trial . Am. Surg. 1990; 56: 71-5.

20. Luukkonen, P.; Jarvinen, H.J.; Haapiainen, R.: Early experience with biofragmentable anastomosis ring in colon surgery. Acta Chir. Scand. 1990; 156: 795-9.

21. Chua, C.L.: Colonic anastomosis with sutureless biofragmentable rings. Singap. Med. J. 1993; 34: 541-4.

22. Irvin, T.T. \& Goligher, J.C.; Johnston, D.: A randomized prospective clinical trial of single-layer and two-layer inverting intestinal anastomoses. Br. J. Surg. 1973; 60: 457-60.

23. Everett, W.G.: A comparison of one layer and two layer techniques for colorectal anastomosis. Br. J. Surg. 1975; 62: $135-40$.

24. Goligher, J.C.; Lee, P.W.G.; Simpkins,K.C. et al : A controlled comparison of one and two-layer techniques of suture for high and low colorectal anastomoses. Br J. Surg. 1977; 64: 609-14.

25. Miller, J.M. : Evalution of a new surgical suture (prolene) . Am. Surg. 1973; 39: 31-9.

26. Hastings, J.C.; Winkle JR., W.V.; Barker, E. et al : Effect of suture materials on healing wounds of the stomach and colon . Surg. Gynecol. Obstet. 1975; 140: 701-7.

27. Edlich, R.F.; Panek, P.H.; Rodeheaver, G.T. et al : Physical and chemical configuration of sutures in the development of surgical infection. Ann. Surg. 1973; 177: 679-88.

28. Pihl, E.; Hughes, E.S.R.; Mcdermontt, F.T. et al: Recurrence of carcinoma of the colon and rectum at the anastomotic suture line. Surg. Gynecol. Obstet. 1981; 153: 495-6.

29. McGregor, J.R.; Galloway, D.J.; Mcculloch, P. et al L: Anastomotic suture materials and implantation metastasis: an experimental study. Br. J. Surg. 1989; 76: 331-4.

30. McCue, J.L. \& Phillips, R.K.S. : Sutureless intestinal anastomoses . Br. J. Surg. 1991; 78: 1291-6.
31. Konishi, F.; Saito,Y.; Ugajin,H. et al : Sutureless anastomosis using a biofragmentable anastomosis ring. Surg. Today 1995; 25: 783-9.

32. Maney, J.W.; Katz,A.R.; Li, L.K. et al: Biofragmentable bowel anastomosis ring : comparative efficacy studies in dogs. Surgery 1988; 103: 56-62.

33. Forde, K.A.; Mclarty, A.J.; Tsai, J. et al: Murphy's button revisited. Clinical experience with the biofragmentable anastomotic ring . Ann. Surg. 1993; 217: 78-81.

34. Wood, J.S. \& Frost, D.B.: Results using the biofragmentable anastomotic ring for colon anastomosis . Am. Surg. 1993; 59: 642-4.

35. Habr-Gama, A.; Soares, J.H.; Campos, F.G.M.C. et al: Anastomose intestinal sem sutura - Resultado inicial com o anel biofragmentável (Valtrac). Rev. Bras. Colo-Proct. 1994; 14: 225-30.

36. Seow-Choe, F. \& Eu, K.W.: Circular staplers versus the biofragmentable ring for colorectal anastomosis: a prospective randomized study . Br. J. Surg. 1994; 81: 178990.

37. Beling, C.A.: Single layer end-to-end intestinal anastomosis. Am. J. Gastroenterol. 1957; 27: 374-80.

38. Bronwell, A.W.; Rutledge,R.; Dalton, M.L.: Single-layer open gastrointestinal anastomosis. Ann.Surg. 1967; 165: 925-32.

39. Peters, H.E. \& Stanten, A.: Aseptic Single Layer Anastomosis of the Bowel: a review of one hundred seventy-five cases. Am. J. Surg. 1971; 122: 159-63.

40. Mantovani, M.; Medeiros, R.R.; Leonardi, L.S.: Sutura seromuscular extramucosa em plano único na cirurgia do intestino. Rev. Paul. Med. 1975; 86: 125-30.

41. Rosenberg, D.; Nasser, A.; Behmer, O.A. et al : Estudo clínico e experimental comparativo de suturas intestinais em plano único com fio de ácido poliglicólico simples e com corante verde. Rev. Paul. Med. 1975; 85: 59-65.

42. Matheson, N.A.; Mcintosh, C.A.; Krukowski, Z.H.: Continuing experience with single layer appositional anastomosis in the large bowel. Br. J. Surg. 1985; 72(sup$\mathrm{pl})$ : 104-6.

43. Carty, N.J.; Keating, J.; Campbell, J.et al: Prospective audit of an extramucosal technique for intestinal anastomosis. Br. J. Surg 1991; 78: 1439-41.

44. Gullichsen, R.; Ovaska, J.; Havia, T. et al: What happens to the Valtrac anastomosis of the colon? A follow-up study. Dis. Colon Rectum 1993; 36: 362-5.

\section{ENDEREÇOPARACORRESPONDÊNCIA}

Dr. Sarhan Sydney Saad

Rua dos Otonis, 861 - Vila Clementino

04025-002 — São Paulo-SP 